\title{
Brouwer Invariance of Domain Theorem ${ }^{1}$
}

\author{
Karol Pąk \\ Institute of Informatics \\ University of Białystok \\ Sosnowa 64, 15-887 Białystok \\ Poland
}

\begin{abstract}
Summary. In this article we focus on a special case of the Brouwer invariance of domain theorem. Let us $A, B$ be a subsets of $\mathcal{E}^{n}$, and $f: A \rightarrow B$ be a homeomorphic. We prove that, if $A$ is closed then $f$ transform the boundary of $A$ to the boundary of $B$; and if $B$ is closed then $f$ transform the interior of $A$ to the interior of $B$. These two cases are sufficient to prove the topological invariance of dimension, which is used to prove basic properties of the $n$-dimensional manifolds, and also to prove basic properties of the boundary and the interior of manifolds, e.g. the boundary of an $n$-dimension manifold with boundary is an $(n-1)$-dimension manifold. This article is based on [18; 21] and [20] can also serve as reference books.
\end{abstract}

MSC: 54A05 03B35

Keywords: continuous transformations; topological dimension

MML identifier: BROUWER3, version: 8.1.02 5.22.1199

The notation and terminology used in this paper have been introduced in the following articles: [27], [1], [14], 44, [6], [15], [37], [7], 8], [40], 31], [34], 38], [2], [3], 9], 5], 33], 13], 44], 45], 10], [42, [43], 35], 17], 28], 29], 25], 46], [16], [47], 26], 30], 32], and [12].

\section{Preliminaries}

From now on $x, X$ denote sets, $n, m, i$ denote natural numbers, $p, q$ denote points of $\mathcal{E}_{\mathrm{T}}^{n}, A, B$ denote subsets of $\mathcal{E}_{\mathrm{T}}^{n}$, and $r, s$ denote real numbers.

Let us consider $X$ and $n$. One can verify that every function from $X$ into $\mathcal{E}_{\mathrm{T}}^{n}$ is finite sequence-yielding.

\footnotetext{
${ }^{1}$ The paper has been financed by the resources of the Polish National Science Centre granted by decision no DEC-2012/07/N/ST6/02147.

(C) 2014 University of Białystok CC-BY-SA License ver. 3.0 or later ISSN 1426-2630(Print), 1898-9934(Online)
} 
Let us consider $m$. Let $f$ be a function from $X$ into $\mathcal{E}_{\mathrm{T}}^{n}$ and $g$ be a function from $X$ into $\mathcal{E}_{\mathrm{T}}^{m}$. Let us observe that the functor $f \frown g$ yields a function from $X$ into $\mathcal{E}_{\mathrm{T}}^{n+m}$. Let $T$ be a topological space. Let $f$ be a continuous function from $T$ into $\mathcal{E}_{\mathrm{T}}^{n}$ and $g$ be a continuous function from $T$ into $\mathcal{E}_{\mathrm{T}}^{m}$. Note that $f \frown g$ is continuous as a function from $T$ into $\mathcal{E}_{\mathrm{T}}^{n+m}$.

Let $f$ be a real-valued function. The functor $|[f]|$ yielding a function is defined by

(Def. 1) (i) $\operatorname{dom} i t=\operatorname{dom} f$, and

(ii) for every object $x$ such that $x \in \operatorname{dom}$ it holds it $(x)=|[f(x)]|$.

One can verify that $|[f]|$ is (the carrier of $\mathcal{E}_{\mathrm{T}}^{1}$ )-valued.

Let us consider $X$. Let $Y$ be a non empty real-membered set and $f$ be a function from $X$ into $Y$. One can verify that the functor $|[f]|$ yields a function from $X$ into $\mathcal{E}_{\mathrm{T}}^{1}$. Let $T$ be a non empty topological space and $f$ be a continuous function from $T$ into $\mathbb{R}^{\mathbf{1}}$. Note that $|[f]|$ is continuous as a function from $T$ into $\mathcal{E}_{\mathrm{T}}^{1}$.

Let $f$ be a continuous real map of $T$. Observe that $|[f]|$ is continuous as a function from $T$ into $\mathcal{E}_{\mathrm{T}}^{1}$.

\section{A Distribution of Sphere}

In the sequel $N$ denotes a non zero natural number and $u, t$ denote points of $\mathcal{E}_{\mathrm{T}}^{N+1}$.

Now we state the propositions:

(1) Let us consider an element $F$ of $\left(\left(\text { the carrier of } \mathbb{R}^{\mathbf{1}}\right)^{\alpha}\right)^{N}$. Suppose If $i \in \operatorname{dom} F$, then $F(i)=\operatorname{PROJ}(N+1, i)$. Then

(i) for every $t,\left(\prod^{*} F\right)(t)=t \uparrow N$, and

(ii) for every subsets $S_{3}, S_{2}$ of $\mathcal{E}_{\mathrm{T}}^{N+1}$ such that $S_{3}=\{u: u(N+1) \geqslant$ 0 and $|u|=1\}$ and $S_{2}=\{t: t(N+1) \leqslant 0$ and $|t|=1\}$ holds $\left(\Pi^{*} F\right)^{\circ} S_{3}=\overline{\operatorname{Ball}}\left(0_{\mathcal{E}_{\mathrm{T}}^{N}}, 1\right)$ and $\left(\Pi^{*} F\right)^{\circ} S_{2}=\overline{\operatorname{Ball}}\left(0_{\mathcal{E}_{\mathrm{T}}^{N}}, 1\right)$ and $\left(\Pi^{*} F\right)^{\circ}\left(S_{3} \cap S_{2}\right)=\operatorname{Sphere}\left(0_{\mathcal{E}_{\mathrm{T}}^{N}}, 1\right)$ and for every function $H$ from $\mathcal{E}_{\mathrm{T}}^{N+1}\left\lceil S_{3}\right.$ into $\operatorname{Tdisk}\left(0_{\mathcal{E}_{\mathrm{T}}^{N}}, 1\right)$ such that $H=\prod^{*} F\left\lceil S_{3}\right.$ holds $H$ is a homeomorphism and for every function $H$ from $\mathcal{E}_{\mathrm{T}}^{N+1} \uparrow S_{2}$ into $\operatorname{Tdisk}\left(0_{\mathcal{E}_{\mathrm{T}}^{N}}, 1\right)$ such that $H=\prod^{*} F\left\lceil S_{2}\right.$ holds $H$ is a homeomorphism, where $\alpha$ is the carrier of $\mathcal{E}_{\mathrm{T}}^{N+1}$. Proof: Set $N_{2}=N+1$. Set $T_{10}=\mathcal{E}_{\mathrm{T}}^{N_{2}}$. Set $T_{4}=\mathcal{E}_{\mathrm{T}}^{N}$. Set $N_{3}=N$ NormF. Set $N_{4}=N_{3} \cdot N_{3}$. Reconsider $O=1$ as an element of $\mathbb{N}$. Set $T_{3}=\operatorname{Tdisk}\left(0_{\mathcal{E}_{\mathrm{T}}^{N}}, 1\right)$. Reconsider $m_{2}=-N_{4}$ as a function from $T_{4}$ into $\mathbb{R}^{\mathbf{1}}$. Reconsider $m_{1}=1+m_{2}$ as a function from $T_{4}$ into $\mathbb{R}^{1}$. Set $F_{1}=\prod^{*} F$. For every $t,\left(\prod^{*} F\right)(t)=t \uparrow N$ by [2, (13)], [41, (25)], [4, (1)]. $\overline{\operatorname{Ball}}\left(0_{T_{4}}, 1\right) \subseteq F_{1}{ }^{\circ} S_{3}$ by [14, (22)], [28, (11)], [6, (16)], 
[11, (145)]. $\overline{\operatorname{Ball}}\left(0_{T_{4}}, 1\right) \subseteq F_{1}{ }^{\circ} S_{2}$ by [14, (22)], [28, (11)], [6, (16)], [11, (145)]. Sphere $\left(0_{T_{4}}, 1\right) \subseteq F_{1}{ }^{\circ}\left(S_{2} \cap S_{3}\right)$ by [14, (22)], [28, (12)], 66, (16), (92)]. $F_{1}{ }^{\circ} S_{3} \subseteq \overline{\operatorname{Ball}}\left(0_{T_{4}}, 1\right)$ by [14, (22)], [4, (59)], [24, (17)], [19, (10)]. $F_{1}{ }^{\circ} S_{2} \subseteq \overline{\operatorname{Ball}}\left(0_{T_{4}}, 1\right)$ by [14, (22)], [4, (59)], [24, (17)], [19, (10)]. $F_{1}{ }^{\circ}\left(S_{2} \cap\right.$ $\left.S_{3}\right) \subseteq$ Sphere $\left(0_{T_{4}}, 1\right)$ by [14, (22)], [4, (59)], [24, (17)], [19, (10)]. For every function $H$ from $\mathcal{E}_{\mathrm{T}}^{N+1}\left\lceil S_{3} \operatorname{into} \operatorname{Tdisk}\left(0_{\mathcal{E}_{\mathrm{T}}^{N}}, 1\right)\right.$ such that $H=\prod^{*} F\left\lceil S_{3}\right.$ holds $H$ is a homeomorphism by [24, (17)], [17, (17)], [2, (11)], [25, (13)]. For every objects $x_{1}, x_{2}$ such that $x_{1}, x_{2} \in \operatorname{dom} H$ and $H\left(x_{1}\right)=H\left(x_{2}\right)$ holds $x_{1}=x_{2}$ by [14, (22)], [19, (10)], [7, (47)], [39, (40)]. Set $T_{3}=\operatorname{Tdisk}\left(0_{T_{4}}, 1\right)$. Set $M=m_{1} \uparrow T_{3}$. Reconsider $M_{1}=M$ as a continuous function from $T_{3}$ into $\mathbb{R}$. Reconsider $M_{2}=-\sqrt{M_{1}}$ as a function from $T_{3}$ into $\mathbb{R}$. For every point $p$ of $T_{4}$ such that $p \in$ the carrier of $T_{3}$ holds $M_{1}(p)=1-|p| \cdot|p|$ by [7, (49)]. Reconsider $S_{1}=\left|\left[M_{2}\right]\right|$ as a continuous function from $T_{3}$ into $\mathcal{E}_{\mathrm{T}}^{1}$. Reconsider $I_{3}=\mathrm{id}_{T_{3}}$ as a continuous function from $T_{3}$ into $T_{4}$. Reconsider $I_{4}=I_{3} \frown S_{1}$ as a continuous function from $T_{3}$ into $\mathcal{E}_{\mathrm{T}}^{N+O}$. For every objects $y, x, y \in \operatorname{rng} H$ and $x=I_{4}(y)$ iff $x \in \operatorname{dom} H$ and $y=H(x)$ by [7, (17)], [11, (145), (144), (55)]. For every subset $P$ of $T_{10}\left\lceil S_{2}, P\right.$ is open iff $H^{\circ} P$ is open by [4, (1)], [2, (13)], [25, (57)].

(2) Let us consider subsets $S_{3}, S_{2}$ of $\mathcal{E}_{\mathrm{T}}^{n}$. Suppose

(i) $S_{3}=\left\{s\right.$, where $s$ is a point of $\mathcal{E}_{\mathrm{T}}^{n}: s(n) \geqslant 0$ and $\left.|s|=1\right\}$, and

(ii) $S_{2}=\left\{t\right.$, where $t$ is a point of $\mathcal{E}_{\mathrm{T}}^{n}: t(n) \leqslant 0$ and $\left.|t|=1\right\}$.

Then

(iii) $S_{3}$ is closed, and

(iv) $S_{2}$ is closed.

(3) Let us consider a metrizable topological space $T_{2}$. Suppose $T_{2}$ is finiteind and second-countable. Let us consider a closed subset $F$ of $T_{2}$. Suppose ind $F^{\mathrm{c}} \leqslant n$. Let us consider a continuous function $f$ from $T_{2}\lceil F$ into TopUnitCircle $(n+1)$. Then there exists a continuous function $g$ from $T_{2}$ into TopUnitCircle $(n+1)$ such that $g\lceil F=f$. Proof: Define $\mathcal{P}$ [natural number] $\equiv$ for every metrizable topological space $T_{2}$ such that $T_{2}$ is finite-ind and second-countable for every closed subset $F$ of $T_{2}$ such that ind $F^{\mathrm{c}} \leqslant \$_{1}$ for every continuous function $f$ from $T_{2} \uparrow F$ into TopUnitCircle $\left(\$_{1}+1\right)$, there exists a function $g$ from $T_{2}$ into TopUnitCircle $\left(\$_{1}+1\right)$ such that $g$ is continuous and $g\lceil F=f$. For every $n$ such that $\mathcal{P}[n]$ holds $\mathcal{P}[n+1]$ by (2), [29, (9)], [42, (13)], [44, (121)]. $\mathcal{P}[(0$ qua natural number)] by [44, (143), (135)], [29, (9)], [14, (70)]. For every $n, \mathcal{P}[n]$ from [2, Sch. 2].

(4) Suppose $p \notin A$ and $r>0$. Then there exists a function $h$ from $\mathcal{E}_{\mathrm{T}}^{n}\lceil A$ into $\mathcal{E}_{\mathrm{T}}^{n} \uparrow \operatorname{Sphere}(p, r)$ such that

(i) $h$ is continuous, and 
(ii) $h \uparrow \operatorname{Sphere}(p, r)=\mathrm{id}_{A \cap \operatorname{Sphere}(p, r)}$.

(5) If $r+|p-q| \leqslant s$, then $\operatorname{Ball}(p, r) \subseteq \operatorname{Ball}(q, s)$.

(6) If $A$ is not boundary, then ind $A=n$.

Now we state the proposition:

(7) The Small Inductive Dimension of the Sphere:

If $r>0$, then ind Sphere $(p, r)=n-1$. Proof: If ind $A \leqslant i$ and ind $B \leqslant i$ and $A$ is closed, then $\operatorname{ind}(A \cup B) \leqslant i$ by [33, (31)], [23, (93)], [35, (22)], [36. (5)].

\section{A Characterization of Open Sets in Euclidean Space in Terms of Continuous Transformations}

Now we state the propositions:

(8) Suppose $n>0$ and $p \in A$ and for every $r$ such that $r>0$ there exists an open subset $U$ of $\mathcal{E}_{\mathrm{T}}^{n} \uparrow A$ such that $p \in U$ and $U \subseteq \operatorname{Ball}(p, r)$ and for every function $f$ from $\mathcal{E}_{\mathrm{T}}^{n} \uparrow(A \backslash U)$ into TopUnitCircle $n$ such that $f$ is continuous there exists a function $h$ from $\mathcal{E}_{\mathrm{T}}^{n} \uparrow A$ into TopUnitCircle $n$ such that $h$ is continuous and $h \uparrow(A \backslash U)=f$. Then $p \in \operatorname{Fr} A$. Proof: Set $T_{7}=\mathcal{E}_{\mathrm{T}}^{n}$. Set $c_{1}=$ the carrier of $T_{7}$. Set $S=$ Sphere $\left(0_{T_{7}}, 1\right)$. Set $T_{9}=$ TopUnitCircle $n$. Reconsider $c=c_{1} \backslash\left\{0_{T_{7}}\right\}$ as a non empty open subset of $T_{7}$. Set $n_{3}=$ $n$ NormF. Set $T_{8}=T_{7}\left\lceil c\right.$. Set $G=\operatorname{transl}\left(p, T_{7}\right)$. Reconsider $I=\stackrel{T_{8}}{\hookrightarrow}$ as a continuous function from $T_{8}$ into $T_{7} .0 \notin \operatorname{rng}\left(n_{3}\left\lceil T_{8}\right)\right.$ by [44, (57)], [14, (22)], [7, (47)], [14, (8), (70)]. Reconsider $n_{2}=n_{3}\left\lceil T_{8}\right.$ as a non-empty continuous function from $T_{8}$ into $\mathbb{R}^{\mathbf{1}}$. Reconsider $b=I / n_{2}$ as a function from $T_{8}$ into $T_{7}$. Set $E_{1}=\mathcal{E}^{n}$. Set $T_{2}=E_{1 \text { top }}$. Reconsider $e=p$ as a point of $E_{1}$. Reconsider $I_{1}=\operatorname{Int} A$ as a subset of $T_{2}$. Consider $r$ being a real number such that $r>0$ and $\operatorname{Ball}(e, r) \subseteq I_{1}$. Set $r_{2}=\frac{r}{2}$. Consider $U$ being an open subset of $T_{7}\left\lceil A\right.$ such that $p \in U$ and $U \subseteq \operatorname{Ball}\left(p, r_{2}\right)$ and for every function $f$ from $T_{7}\left\lceil(A \backslash U)\right.$ into $T_{9}$ such that $f$ is continuous there exists a function $h$ from $T_{7}\left\lceil A\right.$ into $T_{9}$ such that $h$ is continuous and $h \uparrow(A \backslash U)=f$. Reconsider $S_{4}=\operatorname{Sphere}\left(p, r_{2}\right)$ as a non empty subset of $T_{7}$. Consider $a$ being an object such that $a \in S_{4}$. Reconsider $C_{2}=\overline{\operatorname{Ball}}\left(p, r_{2}\right)$ as a non empty subset of $T_{7}$. Reconsider $s_{2}=S_{4}$ as a non empty subset of $T_{7}\left\lceil C_{2}\right.$. Reconsider $A_{1}=A \backslash U$ as a non empty subset of $T_{7}$. Set $T_{1}=T_{7}\left\lceil A_{1}\right.$. Set $t=\operatorname{transl}\left(-p, T_{7}\right)$. Set $T=t\left\lceil T_{1} . \operatorname{rng} T \subseteq c\right.$ by [7, (47)], [42, (21)]. Reconsider $T_{1} 1=T$ as a continuous function from $T_{1}$ into $T_{8}$. For every point $p$ of $T_{7}$ such that $p \in c$ holds $b(p)=\frac{1}{|p|} \cdot p$ and $\left|\frac{1}{|p|} \cdot p\right|=1$ by [22, (84)], [7. (49)], [26, (72)], [12, (56)]. rng $b \subseteq S$ by [42, (13)]. Reconsider $B=b$ as a function from $T_{8}$ into $T_{9}$. Set $m=r_{2} \bullet T_{7}$. Set $M=m\left\lceil T_{9}\right.$. Reconsider $M=m\left\lceil T_{9}\right.$ as a continuous function from $T_{9}$ into $T_{7}$. Reconsider $c_{2}=C_{2}$ as a subset of $T_{7} \uparrow A$. Consider $h$ being a function from $T_{7} \uparrow A$ into $T_{9}$ such 
that $h$ is continuous and $h \uparrow(A \backslash U)=B \cdot T_{1} 1$. Reconsider $G_{2}=G \cdot(M \cdot h)$ as a continuous function from $T_{7}\left\lceil A\right.$ into $T_{7} \cdot \operatorname{rng} G_{2} \subseteq S_{4}$ by [7, (12), (11), (47)], [42, (28), (15)]. Reconsider $g_{2}=G_{2}$ as a function from $T_{7}\lceil A$ into $T_{7}\left\lceil S_{4}\right.$. Reconsider $g_{1}=g_{2} \uparrow\left(\left(T_{7}\lceil A)\left\lceil c_{2}\right)\right.\right.$ as a continuous function from $T_{7}\left\lceil C_{2}\right.$ into $\left(T_{7}\left\lceil C_{2}\right)\left\lceil s_{2}\right.\right.$. For every point $w$ of $T_{7}\left\lceil C_{2}\right.$ such that $w \in S_{4}$ holds $g_{1}(w)=w$ by [7, (11), (12)], [44, (61)], [7, (47)].

(9) Suppose $p \in \operatorname{Fr} A$ and $A$ is closed. Suppose $r>0$. Then there exists an open subset $U$ of $\mathcal{E}_{\mathrm{T}}^{n} \uparrow A$ such that

(i) $p \in U$, and

(ii) $U \subseteq \operatorname{Ball}(p, r)$, and

(iii) for every function $f$ from $\mathcal{E}_{\mathrm{T}}^{n} \uparrow(A \backslash U)$ into TopUnitCircle $n$ such that $f$ is continuous there exists a function $h$ from $\mathcal{E}_{\mathrm{T}}^{n} \uparrow A$ into TopUnitCircle $n$ such that $h$ is continuous and $h \uparrow(A \backslash U)=f$.

Proof: $n>0$ by [14, (77), (22)], [12, (33)]. Set $r_{3}=\frac{r}{3}$. Set $r_{2}=2 \cdot r_{3}$. Set $B=\operatorname{Ball}\left(p, r_{3}\right)$. Consider $x$ being an object such that $x \in A^{\mathrm{c}}$ and $x \in B$. Set $u=\operatorname{Ball}\left(x, r_{2}\right) . u \subseteq \operatorname{Ball}(p, r)$.

\section{Brouwer Invariance of Domain Theorem - Special Case}

Let us consider a function $h$ from $\mathcal{E}_{\mathrm{T}}^{n}\left\lceil A\right.$ into $\mathcal{E}_{\mathrm{T}}^{n}\lceil B$. Now we state the propositions:

(10) If $A$ is closed and $p \in \operatorname{Fr} A$, then if $h$ is a homeomorphism, then $h(p) \in$ Fr $B$. The theorem is a consequence of (9) and (8).

(11) If $B$ is closed and $p \in \operatorname{Int} A$, then if $h$ is a homeomorphism, then $h(p) \in$ Int $B$. The theorem is a consequence of (8) and (9).

(12) Suppose $A$ is closed and $B$ is closed. Then if $h$ is a homeomorphism, then $h^{\circ}(\operatorname{Int} A)=\operatorname{Int} B$ and $h^{\circ}(\operatorname{Fr} A)=\operatorname{Fr} B$. Proof: $h^{\circ}(\operatorname{Int} A)=\operatorname{Int} B$ by (11), (10), [46, (39)].

\section{Topological Invariance of Dimension - An Introduction to MANIFOLDS}

Now we state the proposition:

(13) Suppose $r>0$. Let us consider a subset $U$ of $\operatorname{Tdisk}(p, r)$. Suppose $U$ is open and non empty. Let us consider a subset $A$ of $\mathcal{E}_{\mathrm{T}}^{n}$. If $A=U$, then Int $A$ is not empty.

Let us consider a non empty topological space $T$, subsets $A, B$ of $T, r, s$, a point $p_{1}$ of $\mathcal{E}_{\mathrm{T}}^{n}$, and a point $p_{2}$ of $\mathcal{E}_{\mathrm{T}}^{m}$.

Let us assume that $r>0$ and $s>0$. Now we state the propositions: 
(14) Suppose $T\left\lceil A\right.$ and $T \operatorname{disk}\left(p_{1}, r\right)$ are homeomorphic and $T\left\lceil B\right.$ and $\operatorname{Tdisk}\left(p_{2}, s\right)$ are homeomorphic and Int $A$ meets Int $B$. Then $n=$ $m$. The theorem is a consequence of (13) and (6).

(15) Suppose $T\left\lceil A\right.$ and $\mathcal{E}_{\mathrm{T}}^{n} \uparrow \operatorname{Ball}\left(p_{1}, r\right)$ are homeomorphic and $T\left\lceil B\right.$ and $\operatorname{Tdisk}\left(p_{2}, s\right)$ are homeomorphic and Int $A$ meets Int $B$. Then $n=$ $m$. The theorem is a consequence of (13) and (6).

Now we state the propositions:

(i) $\left(\operatorname{transl}\left(p, \mathcal{E}_{\mathrm{T}}^{n}\right)\right)^{\circ}(\operatorname{Ball}(q, r))=\operatorname{Ball}(q+p, r)$, and

(ii) $\left(\operatorname{transl}\left(p, \mathcal{E}_{\mathrm{T}}^{n}\right)\right)^{\circ}(\overline{\operatorname{Ball}}(q, r))=\overline{\operatorname{Ball}}(q+p, r)$, and

(iii) $\left(\operatorname{transl}\left(p, \mathcal{E}_{\mathrm{T}}^{n}\right)\right)^{\circ}(\operatorname{Sphere}(q, r))=\operatorname{Sphere}((q+p), r)$.

Proof: Set $T_{5}=\mathcal{E}_{\mathrm{T}}^{n}$. Set $T=\operatorname{transl}\left(p, T_{5}\right) \cdot T^{\circ}(\operatorname{Ball}(q, r))=\operatorname{Ball}(q+p, r)$ by [28, (7)], [42, (27)]. $T^{\circ}(\overline{\operatorname{Ball}}(q, r))=\overline{\operatorname{Ball}}(q+p, r)$ by [28, (8)], [42, (27)]. $T^{\circ}(\operatorname{Sphere}(q, r)) \subseteq \operatorname{Sphere}((q+p), r)$ by [28, (9)].

(17) Suppose $s>0$. Then

(i) $\left(s \bullet \mathcal{E}_{\mathrm{T}}^{n}\right)^{\circ}(\operatorname{Ball}(p, r))=\operatorname{Ball}(s \cdot p, r \cdot s)$, and

(ii) $\left(s \bullet \mathcal{E}_{\mathrm{T}}^{n}\right)^{\circ}(\overline{\operatorname{Ball}}(p, r))=\overline{\operatorname{Ball}}(s \cdot p, r \cdot s)$, and

(iii) $\left(s \bullet \mathcal{E}_{\mathrm{T}}^{n}\right)^{\circ}(\operatorname{Sphere}(p, r))=\operatorname{Sphere}((s \cdot p),(r \cdot s))$.

Proof: Set $T_{5}=\mathcal{E}_{\mathrm{T}}^{n}$. Set $M=s \bullet T_{5} \cdot M^{\circ}(\operatorname{Ball}(p, r))=\operatorname{Ball}(s \cdot p, r \cdot s)$ by [42, (34)], [14, (11)], [28, (7)]. $M^{\circ}(\overline{\operatorname{Ball}}(p, r))=\overline{\operatorname{Ball}}(s \cdot p, r \cdot s)$ by [42, (34)], [14, (11)], 28, (8)]. $M^{\circ}(\operatorname{Sphere}(p, r)) \subseteq \operatorname{Sphere}((s \cdot p),(r \cdot s))$ by [42, (34)], [14, (11)], [28, (9)].

(18) Let us consider a rotation homogeneous additive function $f$ from $\mathcal{E}_{\mathrm{T}}^{n}$ into $\mathcal{E}_{\mathrm{T}}^{n}$. Suppose $f$ is onto. Then

(i) $f^{\circ}(\operatorname{Ball}(p, r))=\operatorname{Ball}(f(p), r)$, and

(ii) $f^{\circ}(\overline{\operatorname{Ball}}(p, r))=\overline{\operatorname{Ball}}(f(p), r)$, and

(iii) $f^{\circ}(\operatorname{Sphere}(p, r))=\operatorname{Sphere}((f(p)), r)$.

Proof: $f^{\circ}(\operatorname{Ball}(p, r))=\operatorname{Ball}(f(p), r)$ by $[28,(7)] . f^{\circ}(\overline{\operatorname{Ball}}(p, r))=$

$\overline{\operatorname{Ball}}(f(p), r)$ by [28, (8)]. $f^{\circ}(\operatorname{Sphere}(p, r)) \subseteq \operatorname{Sphere}((f(p)), r)$ by [28, (9)]. Consider $x$ being an object such that $x \in \operatorname{dom} f$ and $f(x)=y$.

(19) Let us consider points $p, q$ of $\mathcal{E}_{\mathrm{T}}^{n+1}, r$, and $s$. Suppose

(i) $s \leqslant r \leqslant|p-q|$, and

(ii) $s<|p-q|<s+r$.

Then there exists a function $h$ from $\mathcal{E}_{\mathrm{T}}^{n+1} \uparrow(\operatorname{Sphere}(p, r) \cap \overline{\operatorname{Ball}}(q, s))$ into $\operatorname{Tdisk}\left(0_{\mathcal{E}_{\mathrm{T}}^{n}}, 1\right)$ such that

(iii) $h$ is a homeomorphism, and

(iv) $h^{\circ}(\operatorname{Sphere}(p, r) \cap \operatorname{Sphere}(q, s))=\operatorname{Sphere}\left(0_{\mathcal{E}_{\mathrm{T}}^{n}}, 1\right)$. 
Proof: Set $n_{1}=n+1$. Set $T_{6}=\mathcal{E}_{\mathrm{T}}^{n_{1}}$. Set $y=\frac{1}{r} \cdot(q-p)$. Set $Y=$ $\langle\underbrace{0, \ldots, 0}_{n_{1}}\rangle+\cdot\left(n_{1},|y|\right)$. There exists a homogeneous additive rotation function $R$ from $T_{6}$ into $T_{6}$ such that $R$ is a homeomorphism and $R(y)=Y$ by [34, (40), (41)]. Consider $R$ being a homogeneous additive rotation function from $T_{6}$ into $T_{6}$ such that $R$ is a homeomorphism and $R(y)=Y$. $s>0$.

\section{REFERENCES}

[1] Grzegorz Bancerek. Cardinal numbers. Formalized Mathematics, 1(2):377-382, 1990.

[2] Grzegorz Bancerek. The fundamental properties of natural numbers. Formalized Mathematics, 1(1):41-46, 1990.

[3] Grzegorz Bancerek. The ordinal numbers Formalized Mathematics, 1(1):91-96, 1990.

[4] Grzegorz Bancerek and Krzysztof Hryniewiecki. Segments of natural numbers and finite sequences Formalized Mathematics, 1(1):107-114, 1990.

[5] Leszek Borys. Paracompact and metrizable spaces Formalized Mathematics, 2(4):481485, 1991.

[6] Czesław Byliński. Finite sequences and tuples of elements of a non-empty sets Formalized Mathematics, 1(3):529-536, 1990.

[7] Czesław Byliński. Functions and their basic properties Formalized Mathematics, 1(1): 55-65, 1990.

[8] Czesław Byliński. Functions from a set to a set. Formalized Mathematics, 1(1):153-164, 1990.

[9] Czesław Byliński. Partial functions. Formalized Mathematics, 1(2):357-367, 1990.

[10] Czesław Byliński. Introduction to real linear topological spaces. Formalized Mathematics, 13(1):99-107, 2005.

[11] Czesław Byliński. The sum and product of finite sequences of real numbers. Formalized Mathematics, 1(4):661-668, 1990.

[12] Czesław Byliński. Some basic properties of sets. Formalized Mathematics, 1(1):47-53, 1990.

[13] Czesław Byliński and Piotr Rudnicki. Bounding boxes for compact sets in $\mathcal{E}^{2}$. Formalized Mathematics, 6(3):427-440, 1997.

[14] Agata Darmochwał. The Euclidean space Formalized Mathematics, 2(4):599-603, 1991.

[15] Agata Darmochwał. Finite sets Formalized Mathematics, 1(1):165-167, 1990.

[16] Agata Darmochwał. Families of subsets, subspaces and mappings in topological spaces Formalized Mathematıcs, 1(2):257-261, 1990.

[17] Agata Darmochwał and Yatsuka Nakamura. Metric spaces as topological spaces - fundamental concepts. Formalized Mathematics, 2(4):605-608, 1991.

[18] Roman Duda. Wprowadzenie do topologii. PWN, 1986.

[19] Noboru Endou and Yasunari Shidama. Completeness of the real Euclidean space. Formalized Mathematics, 13(4):577-580, 2005.

[20] Ryszard Engelking. Dimension Theory. North-Holland, Amsterdam, 1978.

[21] Ryszard Engelking. General Topology. Heldermann Verlag, Berlin, 1989.

[22] Zbigniew Karno. Continuity of mappings over the union of subspaces. Formalized Mathematics, 3(1):1-16, 1992 .

[23] Zbigniew Karno. Separated and weakly separated subspaces of topological spaces. Formalized Mathematıcs, 2(5):665-674, 1991.

[24] Artur Korniłowicz. Homeomorphism between $\left[: \mathcal{E}_{\mathrm{T}}^{i}, \mathcal{E}_{\mathrm{T}}^{j}:\right]$ and $\mathcal{E}_{\mathrm{T}}^{i+j}$ Formalized Mathematics, 8(1):73-76, 1999.

[25] Artur Korniłowicz. On the continuity of some functions. Formalized Mathematics, 18(3): 175-183, 2010. doi $10.2478 / v 10037-010-0020-z$.

[26] Artur Korniłowicz. Arithmetic operations on functions from sets into functional sets. Formalized Mathematics, 17(1):43-60, 2009. doi 10.2478/v10037-009-0005-y. 
[27] Artur Korniłowicz and Yasunari Shidama. Brouwer fixed point theorem for disks on the plane, Formalized Mathematics, 13(2):333-336, 2005.

[28] Artur Korniłowicz and Yasunari Shidama. Intersections of intervals and balls in $\mathcal{E}_{\mathrm{T}}^{n}$ Formalized Mathematics, 12(3):301-306, 2004.

[29] Artur Korniłowicz and Yasunari Shidama. Some properties of circles on the plane Formalized Mathematics, 13(1):117-124, 2005.

[30] Eugeniusz Kusak, Wojciech Leończuk, and Michał Muzalewski. Abelian groups, fields and vector spaces. Formalized Mathematics, 1(2):335-342, 1990.

[31] Roman Matuszewski and Yatsuka Nakamura. Projections in $n$-dimensional Euclidean space to each coordinates Formalized Mathematıcs, 6(4):505-509, 1997.

[32] Robert Milewski. Bases of continuous lattices. Formalized Mathematics, 7(2):285-294, 1998.

[33] Beata Padlewska and Agata Darmochwał. Topological spaces and continuous functions Formalized Mathematics, 1(1):223-230, 1990.

[34] Karol Pak. The rotation group. Formalized Mathematics, 20(1):23-29, 2012. doi $10.2478 /$ v10037-012-0004-2.

[35] Karol Pąk. Small inductive dimension of topological spaces. Formalized Mathematics, 17 (3):207-212, 2009. doi:10.2478/v10037-009-0025-7

[36] Karol Pąk. Small inductive dimension of topological spaces. Part II. Formalized Mathematics, 17(3):219-222, 2009. doi 10.2478/v10037-009-0027-5

[37] Andrzej Trybulec. Binary operations applied to functions Formalized Mathematics, 1 (2):329-334, 1990.

[38] Andrzej Trybulec. On the sets inhabited by numbers. Formalized Mathematics, 11(4): 341-347, 2003.

[39] Andrzej Trybulec and Czesław Byliński. Some properties of real numbers Formalized Mathematics, 1(3):445-449, 1990.

[40] Michał J. Trybulec. Integers Formalized Mathematics, 1(3):501-505, 1990.

[41] Wojciech A. Trybulec. Non-contiguous substrings and one-to-one finite sequences. Formalized Mathematics, 1(3):569-573, 1990.

[42] Wojciech A. Trybulec. Vectors in real linear space. Formalized Mathematics, 1(2):291-296, 1990.

[43] Zinaida Trybulec. Properties of subsets. Formalized Mathematics, 1(1):67-71, 1990.

[44] Edmund Woronowicz. Relations and their basic properties. Formalized Mathematics, 1 (1):73-83, 1990.

[45] Edmund Woronowicz. Relations defined on sets. Formalized Mathematics, 1(1):181-186, 1990.

[46] Mirosław Wysocki and Agata Darmochwał. Subsets of topological spaces Formalized Mathematics, 1(1):231-237, 1990.

[47] Mariusz Żynel and Adam Guzowski. To topological spaces. Formalized Mathematics, 5 (1):75-77, 1996. 